\title{
Open Mapping Theorem
}

\author{
Hideki Sakurai \\ Hisayoshi Kunimune \\ Yasunari Shidama \\ Shinshu University \\ Shinshu University \\ Shinshu University \\ Nagano, Japan \\ Nagano, Japan \\ Nagano, Japan
}

Summary. In this article we formalize one of the most important theorems of linear operator theory the Open Mapping Theorem commonly used in a standard book such as [8] in chapter 2.4.2. It states that a surjective continuous linear operator between Banach spaces is an open map.

MML identifier: LOPBAN_6, version: $\underline{7.10 .014 .111 .1036}$

The notation and terminology used here are introduced in the following papers: [13], [14], [3], [9], [2], [7], [1], [4], [5], [10], [6], [12], [11], and [15].

The following proposition is true

(1) For all real numbers $x, y$ such that $0 \leq x<y$ there exists a real number $s_{0}$ such that $0<s_{0}$ and $x<\frac{y}{1+s_{0}}<y$.

The scheme RecExD3 deals with a non empty set $\mathcal{A}$, an element $\mathcal{B}$ of $\mathcal{A}$, an element $\mathcal{C}$ of $\mathcal{A}$, and a 4 -ary predicate $\mathcal{P}$, and states that:

There exists a function $f$ from $\mathbb{N}$ into $\mathcal{A}$ such that $f(0)=\mathcal{B}$ and $f(1)=\mathcal{C}$ and for every element $n$ of $\mathbb{N}$ holds $\mathcal{P}[n, f(n), f(n+$ 1), $f(n+2)]$

provided the parameters meet the following requirement:

- For every element $n$ of $\mathbb{N}$ and for all elements $x, y$ of $\mathcal{A}$ there exists an element $z$ of $\mathcal{A}$ such that $\mathcal{P}[n, x, y, z]$.

In the sequel $X, Y$ denote real normed spaces.

The following propositions are true:

(2) For every point $y_{1}$ of $X$ and for every real number $r$ holds Ball $\left(y_{1}, r\right)=$ $y_{1}+\operatorname{Ball}\left(0_{X}, r\right)$.

(3) For every real number $r$ and for every real number $a$ such that $0<a$ holds $\operatorname{Ball}\left(0_{X}, a \cdot r\right)=a \cdot \operatorname{Ball}\left(0_{X}, r\right)$. 
(4) For every linear operator $T$ from $X$ into $Y$ and for all subsets $B_{0}, B_{1}$ of $X$ holds $T^{\circ}\left(B_{0}+B_{1}\right)=T^{\circ} B_{0}+T^{\circ} B_{1}$.

(5) Let $T$ be a linear operator from $X$ into $Y, B_{0}$ be a subset of $X$, and $a$ be a real number. Then $T^{\circ}\left(a \cdot B_{0}\right)=a \cdot T^{\circ} B_{0}$.

(6) Let $T$ be a linear operator from $X$ into $Y, B_{0}$ be a subset of $X$, and $x_{1}$ be a point of $X$. Then $T^{\circ}\left(x_{1}+B_{0}\right)=T\left(x_{1}\right)+T^{\circ} B_{0}$.

(7) For all subsets $V, W$ of $X$ and for all subsets $V_{1}, W_{1}$ of LinearTopSpaceNorm $X$ such that $V=V_{1}$ and $W=W_{1}$ holds $V+W=$ $V_{1}+W_{1}$.

(8) Let $V$ be a subset of $X, x$ be a point of $X, V_{1}$ be a subset of LinearTopSpaceNorm $X$, and $x_{1}$ be a point of LinearTopSpaceNorm $X$. If $V=V_{1}$ and $x=x_{1}$, then $x+V=x_{1}+V_{1}$.

(9) For every subset $V$ of $X$ and for every real number $a$ and for every subset $V_{1}$ of LinearTopSpaceNorm $X$ such that $V=V_{1}$ holds $a \cdot V=a \cdot V_{1}$.

(10) For every subset $V$ of TopSpaceNorm $X$ and for every subset $V_{1}$ of LinearTopSpaceNorm $X$ such that $V=V_{1}$ holds $\bar{V}=\overline{V_{1}}$.

(11) For every point $x$ of $X$ and for every real number $r$ holds Ball $\left(0_{X}, r\right)=$ $(-1) \cdot \operatorname{Ball}\left(0_{X}, r\right)$

(12) For every point $x$ of $X$ and for every real number $r$ and for every subset $V$ of LinearTopSpaceNorm $X$ such that $V=\operatorname{Ball}(x, r)$ holds $V$ is convex.

(13) Let $x$ be a point of $X, r$ be a real number, $T$ be a linear operator from $X$ into $Y$, and $V$ be a subset of LinearTopSpaceNorm $Y$. If $V=T^{\circ} \operatorname{Ball}(x, r)$, then $V$ is convex.

(14) For every point $x$ of $X$ and for all real numbers $r, s$ such that $r \leq s$ holds $\operatorname{Ball}(x, r) \subseteq \operatorname{Ball}(x, s)$.

(15) Let $X$ be a real Banach space, $Y$ be a real normed space, $T$ be a bounded linear operator from $X$ into $Y, r$ be a real number, $B_{2}$ be a subset of LinearTopSpaceNorm $X$, and $T_{1}, B_{3}$ be subsets of LinearTopSpaceNorm $Y$. If $r>0$ and $B_{2}=\operatorname{Ball}\left(0_{X}, 1\right)$ and $B_{3}=\operatorname{Ball}\left(0_{Y}, r\right)$ and $T_{1}=$ $T^{\circ} \operatorname{Ball}\left(0_{X}, 1\right)$ and $B_{3} \subseteq \overline{T_{1}}$, then $B_{3} \subseteq T_{1}$.

(16) Let $X, Y$ be real Banach spaces, $T$ be a bounded linear operator from $X$ into $Y$, and $T_{2}$ be a function from LinearTopSpaceNorm $X$ into LinearTopSpaceNorm $Y$. If $T_{2}=T$ and $T_{2}$ is onto, then $T_{2}$ is open.

\section{REFERENCES}

[1] Grzegorz Bancerek. The fundamental properties of natural numbers. Formalized Mathematics, 1(1):41-46, 1990.

[2] Grzegorz Bancerek. The ordinal numbers. Formalized Mathematics, 1(1):91-96, 1990.

[3] Czesław Byliński. Functions from a set to a set. Formalized Mathematics, 1(1):153-164, 1990.

[4] Noboru Endou, Takashi Mitsuishi, and Yasunari Shidama. Convex sets and convex combinations. Formalized Mathematics, 11(1):53-58, 2003. 
[5] Noboru Endou, Takashi Mitsuishi, and Yasunari Shidama. Dimension of real unitary space. Formalized Mathematics, 11(1):23-28, 2003.

[6] Noboru Endou, Yasunari Shidama, and Katsumasa Okamura. Baire's category theorem and some spaces generated from real normed space. Formalized Mathematics, 14(4):213$219,2006$.

[7] Krzysztof Hryniewiecki. Basic properties of real numbers. Formalized Mathematics, 1(1):35-40, 1990.

[8] Isao Miyadera. Functional Analysis. Riko-Gaku-Sya, 1972.

[9] Beata Padlewska and Agata Darmochwał. Topological spaces and continuous functions. Formalized Mathematics, 1(1):223-230, 1990.

[10] Jan Popiołek. Real normed space. Formalized Mathematics, 2(1):111-115, 1991.

[11] Hideki Sakurai, Hisayoshi Kunimune, and Yasunari Shidama. Uniform boundedness principle. Formalized Mathematics, 16(1):19-21, 2008.

[12] Yasunari Shidama. Banach space of bounded linear operators. Formalized Mathematics, 12(1):39-48, 2004.

[13] Zinaida Trybulec. Properties of subsets. Formalized Mathematics, 1(1):67-71, 1990.

[14] Edmund Woronowicz. Relations defined on sets. Formalized Mathematics, 1(1):181-186, 1990.

[15] Mariusz Żynel and Adam Guzowski. To topological spaces. Formalized Mathematics, 5(1):75-77, 1996.

Received September 23, 2008 